\title{
Adverse events of focused ultrasound surgery for uterine fibroids and adenomyosis
}

This article was published in the following Dove Press journal:

Reports in Medical Imaging

\author{
Nguyen Minh Duc ${ }^{1, *}$ \\ Huynh Quang Huyl,* \\ Bilgin Keserci ${ }^{2,3}$
}

'Department of Radiology, Pham Ngoc Thach University of Medicine, Ho Chi Minh City, Vietnam; ${ }^{2}$ Department of Radiology, School of Medical Sciences, Universiti Sains Malaysia, Kelantan, Malaysia; ${ }^{3}$ Department of Radiology, Hospital Universiti Sains Malaysia, USM 16I50 Kubang Kerian, Kelantan, Malaysia

*These authors contributed equally to this work
Correspondence: Nguyen Minh Duc Department of Radiology, Pham Ngoc Thach University of Medicine, 86/2 Thanh Thai Ward 12 District II Ho Chi Minh City, Vietnam

Tel +8490288 6899

Email bsnguyenminhduc@pnt.edu.vn

\begin{abstract}
Uterine fibroids and adenomyosis are two common gynecological benign tumors that deteriorate women's quality of life. The prevalence of uterine fibroid and adenomyosis is approximately $70 \%-80 \%$ and $5 \%-70 \%$, respectively. The efficacy and safety of focused ultrasound surgery (FUS) therapy for treating uterine fibroid and adenomyosis patients has been proved. Regardless of this fact, there are still some potential adverse events that may arise during and after the provision of FUS treatment, which can degrade patients' quality of life. Understanding the possible adverse events of FUS treatment can improve the confidence in the selection of eligible patients, optimization of treatment strategy, and monitoring of the treatment procedure to ensure patients' safety.
\end{abstract}

Keywords: adenomyosis, leiomyoma, adverse events, focused ultrasound surgery

\section{Introduction}

Uterine fibroids and adenomyosis are two common gynecological benign tumors that lower women's quality of life during their reproductive age. The prevalence of uterine fibroid and adenomyosis is approximately $70 \%-80 \%$ and $5 \%-70 \%$, respectively. ${ }^{1-5}$ Uterine fibroids, also known as leiomyomas, are constituted by smooth muscle tissue and fibrous connective tissue produced by the myometrium. Conversely, adenomyosis represents a problematic medical condition that is characterized by the abnormal presence of an endometrial tissue infiltrating into the myometrial tissue. Both diseases regularly generate symptoms such as abnormal uterine bleeding, bulk effects, and infertility. ${ }^{1-5}$

Focused ultrasound surgery (FUS) represents a non-invasive method that causes tumors' thermal ablation through the localization of a high-intensity sonication beam that generates coagulative necrotic tissues at a focal spot. FUS therapy is an emerging treatment for many ailments: uterine fibroids, adenomyosis, essential tremor, benign hypertrophic prostate, and breast tumor among others. The efficacy and safety of FUS therapy for patients with uterine fibroid or adenomyosis has been proved through research and practice. ${ }^{2-6}$ Owing to the benefits of FUS therapy, previous research works focused more on the treatment's efficacy and experimental models than its possible adverse outcomes. Nonetheless, some plausible adverse events have been reported during and after FUS treatment, which diminished patients' quality of life. Understanding these effects and finding solutions to minimize such events might lead to an increase in the confidence in the selection of eligible patients, optimization of treatment strategy, and monitoring of treatment procedure to ensure patients' safety. 
Adverse events are related to the near-field area (the zone in front of targeted lesion), far-field area (the zone behind the targeted lesion), local targeted area, adjacent organs surrounding the uterus, and whole body. ${ }^{3,4}$ In addition, ablation of leiomyosarcoma was also a severe adverse event caused by misdiagnosis that led to increasing rate of metastasis and mortality owing to delay of precise treatment. Therefore, in this article, we aimed to describe the adverse events of FUS therapy for uterine fibroids and adenomyosis occurring during and after treatment.

\section{Adverse events related to near-field region}

An ultrasonic beam propagates through the skin surface into the abdominal subcutaneous layer, passes through the abdominal skeletal muscle, and reaches the focal spot (Figure 1), and during delivery, these tissues can absorb sonication energy, disperse, and scatter it. ${ }^{7,8}$ One of the early signs of the side effects of heat accumulation in the near-field during the ablation procedure is fat edema as the adipose tissue is less perfused than the skin and muscle; therefore, adipose tissue was insufficient in heat dissipation. ${ }^{7,8}$ Keserci et al reported that three $(9.68 \%)$ of 31 adenomyosis patients and $12(16.22 \%)$ of 74 uterine fibroid patients who were subjected to magnetic resonance imaging (MRI)-guided
FUS therapy, respectively, manifested particularly high signal intensity of the subcutaneous fat tissue, considered as adipose edema post-FUS therapy (Figure 2)..$^{9,10}$ This adverse event produced no symptoms and was resolved naturally without any interventions.

Leon-Villapalos et al reported that a 39-year-old patient with uterine fibroids was admitted to a MRI-guided FUS therapy clinical trial. ${ }^{11}$ On a physical examination at 2-week post-FUS ablation, it was observed that she had a $10 \times 5 \mathrm{~cm}$ full thickness skin burn, regarded as a grade III skin burn, and partial thickness skin burns in some enclosed areas. In addition, MRI unveiled a small hyper-intense spot adjacent to the skin burns within the left rectus muscle; this was considered as muscle edema as the areas were apparently associated with skin burns. The patient felt no sensation at the lesions and the lesions were cured through the eradication of burned areas.

Scars are described as special tissues containing a lesser degree of perfusion than other tissues, which leads to the inefficient dissipation of heat through them. This in turn leads to higher ultrasonic absorption in the scar tissue compared to the surrounding tissues, which can inevitably activate heat accumulation and thermal damage. Additionally, as scar tissues contain a lesser number of nerve endings, their sensation is attenuated in comparison to the surrounding tissues, which

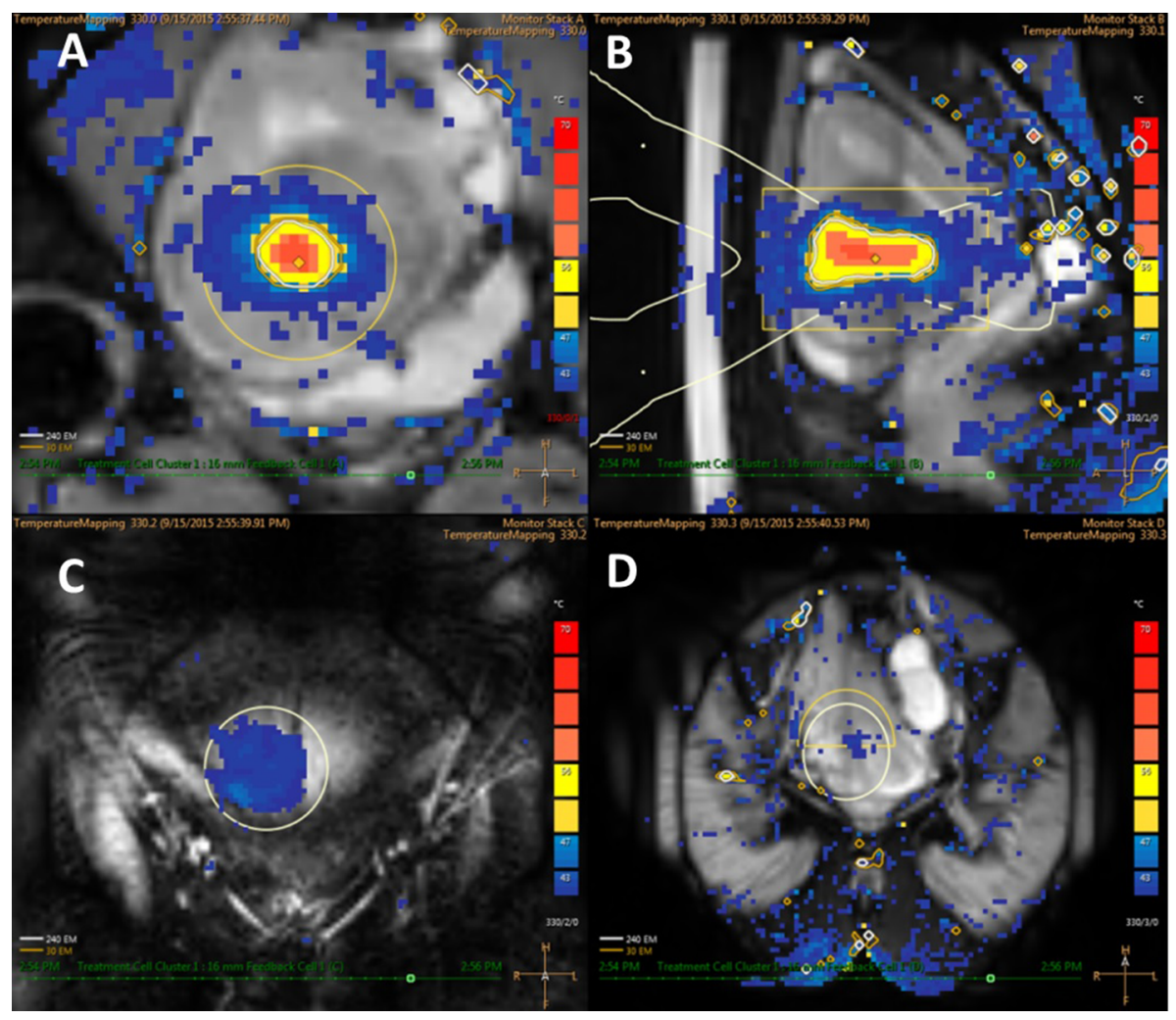

Figure I The temperature map images show (A) coronal plane, (B) sagittal plane, (C) near-field, and (D) far-field. 
can lead clinicians and patients to underestimate the severity of the damage. ${ }^{12}$ In a study conducted by Chen et al in a group of 9,988 patients, in which an ultrasound-guided FUS was adopted, the findings displayed two $(0.02 \%)$ severe skin burn cases out of the $26(0.26 \%)$ skin burn cases, necessitating surgical elimination of the subcutaneous necrotic tissue. ${ }^{13}$ In a recent study by Liu et al, 27,053 patients underwent ultrasound-guided FUS. The results manifested that after ablation, redness of the skin, blisters, and severe skin burns were found in $86(0.32 \%), 20(0.074 \%)$, and $38(0.14 \%)$

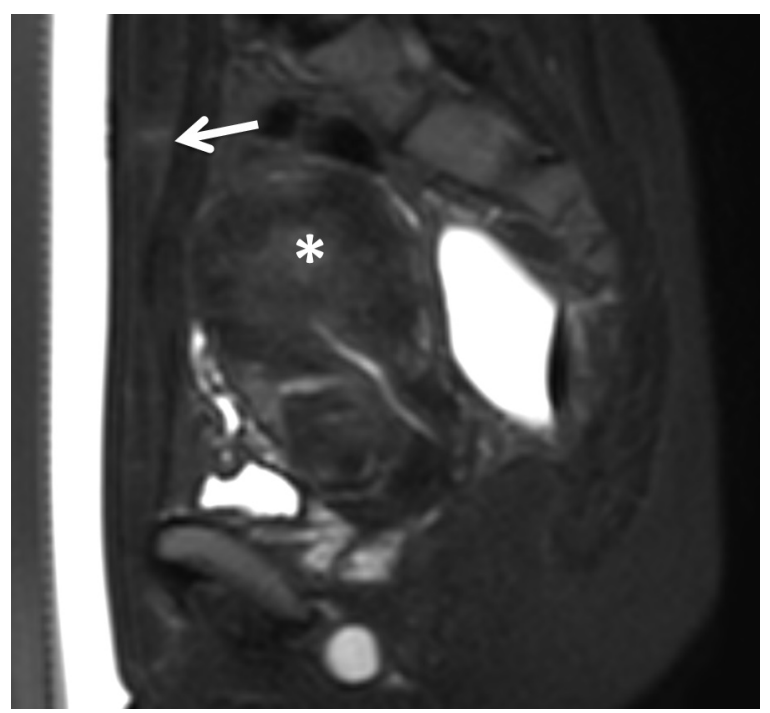

Figure 2 Sagittal fat-saturated T2W image displays an abnormally high-signal intensity region inside a subcutaneous fat layer (white arrow) after focused ultrasound surgery for adenomyosis (white asterisk). patients, respectively. Furthermore, all 38 patients with severe skin burns also had prior abdominal scars owing to older surgeries, and the burned tissues were eliminated through surgical excision. ${ }^{14}$ It was concluded that strict observation during the process of FUS ablation was essential to prevent not only excessive heat accumulation in the near-field, such as fat edema, but also skin burns. ${ }^{9-14}$ Clinicians should consider using the angulation of the transducer, beam shaping (Figure 3), scar patches (Figure 4), or miscellaneous methods (Figure 5 ) to reduce the risk of skin or scar burn. ${ }^{12,15,16}$

Bone tissues facilitate the absorption of sonication energy, augmenting heat accumulation in bones and injuring the skin, the subcutaneous fat layer surrounding this area, and even the bone. ${ }^{6}$ Liu et al reported that after FUS treatment for a cervical leiomyoma, their patient suffered a pubic symphysis burn due to exposure to the sonication beam, which directly penetrated this sensitive region; besides, MRI manifested abnormally high signal intensity of pubis symphysis. ${ }^{14}$ Tilting the transducer to refrain pubis symphysis should be regarded as a fast and important solution to this possible problem. ${ }^{15}$

\section{Adverse events related to far-field region}

After the ablation of the targeted focal spot, a small residual acoustic energy passes through the far-field area where the sciatic nerves, lumbosacral plexus, and sacral bone are located. The nerves and bones can absorb even small amounts of ultrasonic energy, sufficient to cause nerve stimulation and/or bone denaturation (Figure 6). This effect produces

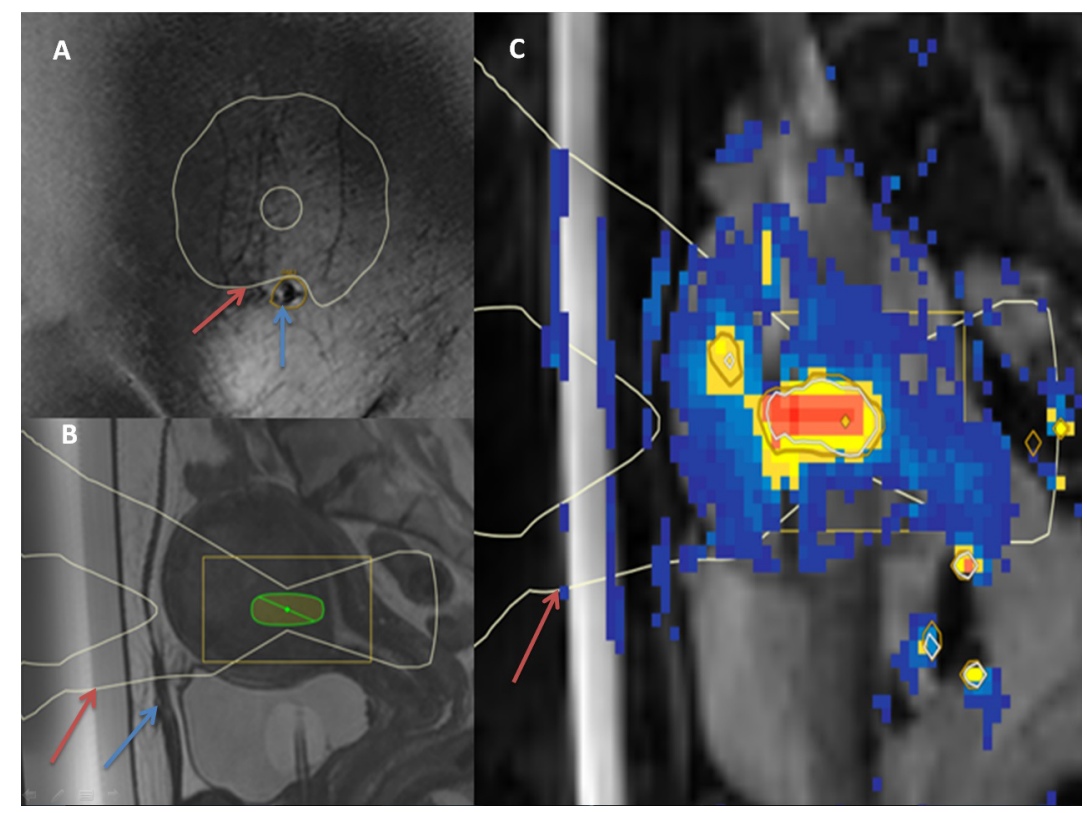

Figure 3 The coronal (A) and sagittal (B) T2W images exhibit the beam shaping (red arrow) and organ avoidance region surrounding the abdominal scar (blue arrow). The sagittal temperature map (C) displays the beam shaping without sonicating the abdominal scar (red arrow). 


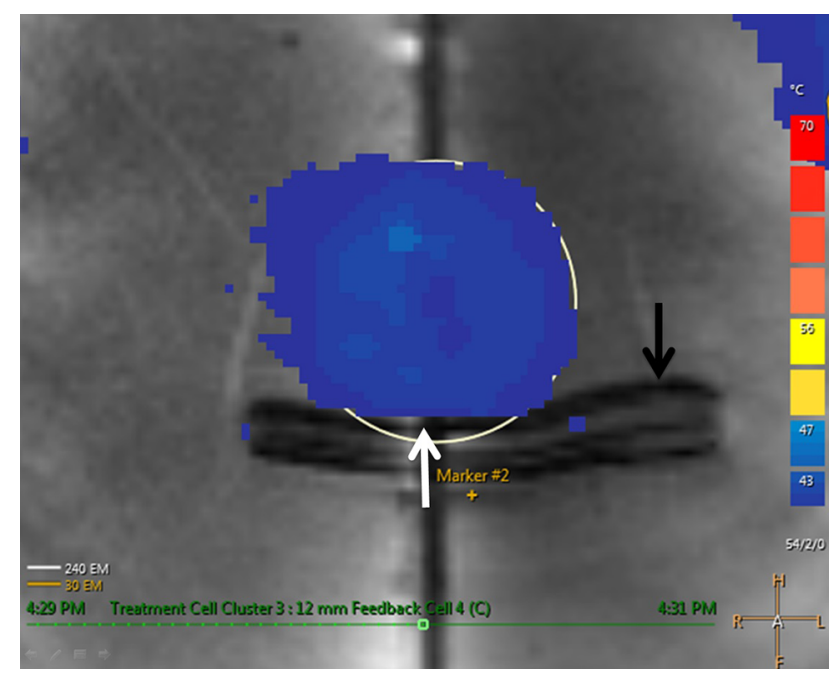

Figure 4 The coronal temperature map displays that the sonication is reflected completely (white arrow) by utilizing scar patch (black arrow).

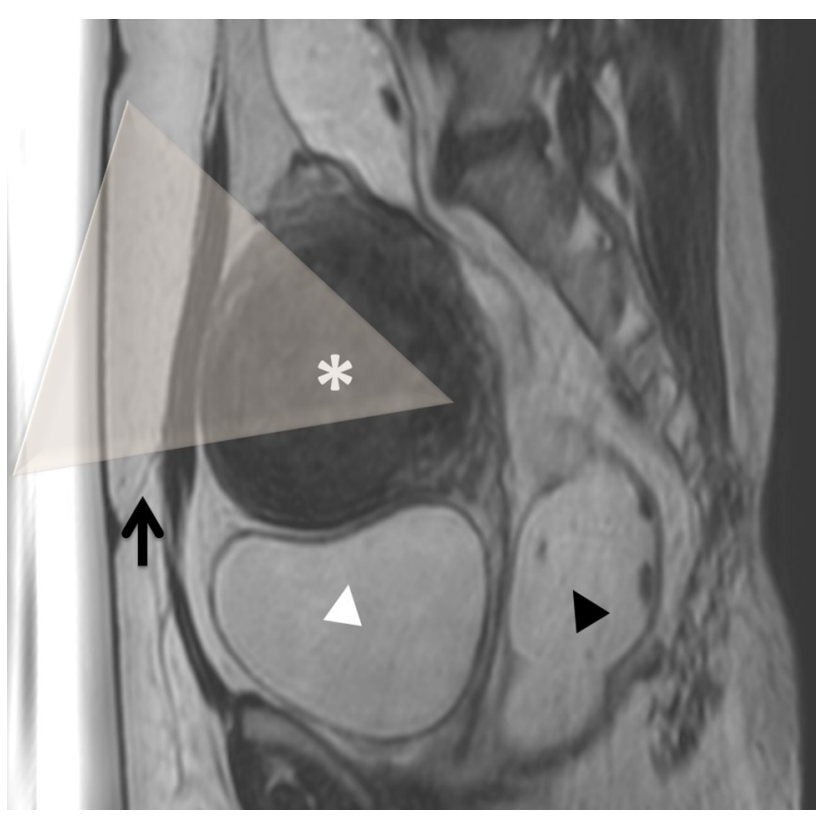

Figure 5 Sagittal T2W images display the uterine fibroid on the anterior wall of the uterus (white asterisk).

Notes: Bladder filled with $400 \mathrm{~mL}$ normal saline (white arrow head) and rectum filled with $200 \mathrm{~mL}$ ultrasound gel (black arrow head) press the uterus to move upward and forward, resulting in the scar position being lower than the tumor position. The tilting transducer leads the beam path and avoids the sonication of the abdominal scar (black arrow).

common symptoms of back, buttock, leg, sacrococcygeal pain, and numbness. ${ }^{9}, 10,13-15,17$

In Hwang et al's study, after the adoption of ultrasoundguided FUS therapy, patients reported severe back pain and computed tomography (CT) results revealed air inside the $\mathrm{T} 5$ to $\mathrm{S} 1$ vertebrae and bone destruction at the L5 vertebra. Moreover, a biopsy under CT guidance was performed, and the histopathological osteolytic samples showed acute inflammatory cell infiltration in the bone marrow and necrotic bone particles. The patient showed total recovery at 3-week post-treatment with antibiotics and painkillers. ${ }^{18}$

The FDA Maude database reported one case of nerve injury due to an undetected motion during MRI-guided FUS therapy, which led to the neglectful sonication of the right lumbosacral plexus. Consequently, the patient was admitted to the emergency unit for 9 days, with the main symptom being the inability to walk and right foot's weakness. The necessary rehabilitation was provided to resolve these symptoms after 3 months. ${ }^{19}$

\section{Adverse events related to local region}

Pelvic pain

Patients commonly reported pain in the pelvic area in spontaneous post-FUS ablation, especially in the region of the targeted tumor. This adverse effect was caused due to a necrotic tumor, local inflammation, and ulceration of the nonperfused tissue (Figure 7), which often disappeared without any medications. ${ }^{6,9,10,13,14}$

\section{Heavy vaginal discharge}

Vaginal discharge occurred commonly after FUS treatment due to the excretion of necrotic tissue through the vagina. ${ }^{6,9,10,13}$ Nevertheless, heavy vaginal discharge should be considered as an adverse event, which can conceivably result in anemia. ${ }^{14}$ Kim et al showed that a 41 -year-old patient with a $5.5 \mathrm{~cm}$ submucosal leiomyoma underwent MRI-guided FUS therapy and reported heavy vaginal discharge, implying that she did not adapt to medications at 8-month post-treatment. Her blood test further exhibited a hemoglobin concentration of $5.0 \mathrm{mIU} / \mathrm{L}$, for which a blood transfusion was performed to increase the hemoglobin concentration up to $9.2 \mathrm{mIU} / \mathrm{L}$, while the MRI showed a $6.1 \mathrm{~cm}$ submucosal leiomyoma, for which a surgery was performed to eradicate the tumor. ${ }^{20}$

\section{Expulsion to uterine cavity}

Jeong et al demonstrated that six (3.82\%) out of 157 transmural uterine fibroids treated with MRI-guided FUS therapy bulged towards the uterine cavity after 5- to 73-day post-FUS ablation, as the uterine fibroid was more diminished in the myometrium part than in the endometrium part, leading to an asymmetrical shape inclined toward the uterine cavity (Figure 8). Hysteroscopic myomectomy represents a useful alternative treatment to resolve this adverse event. ${ }^{21}$ Uterine fibroid shrinkage can transform a transmural uterine fibroid into a submucosal uterine fibroid and finally a pedunculated 

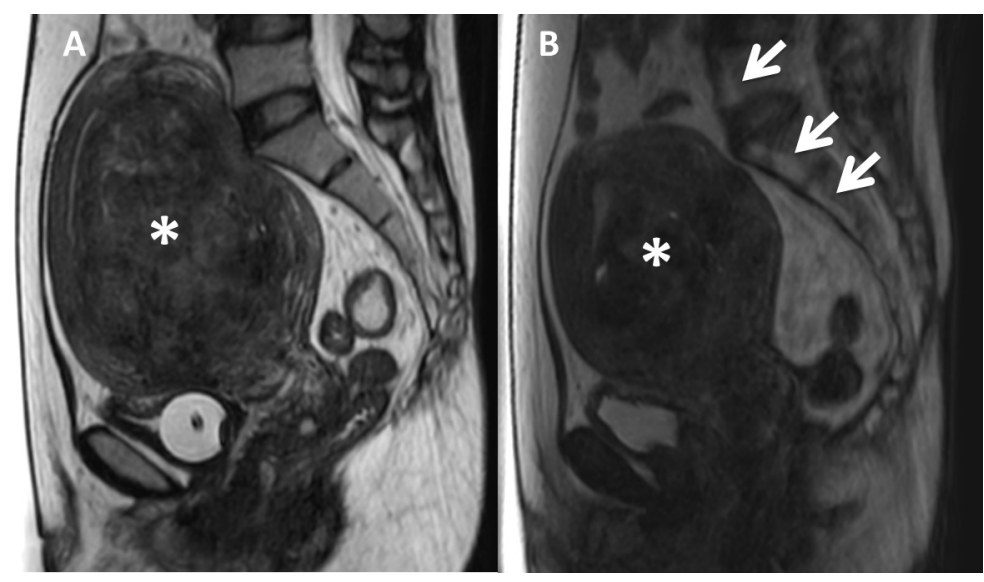

Figure 6 Sagittal T2W images display T5 to S3 vertebrae with normal signal intensity (A) before treatment and abnormal high signal intensity (B) due to bone degeneration (white arrow) after focused ultrasound surgery for uterine fibroid (white asterisk).

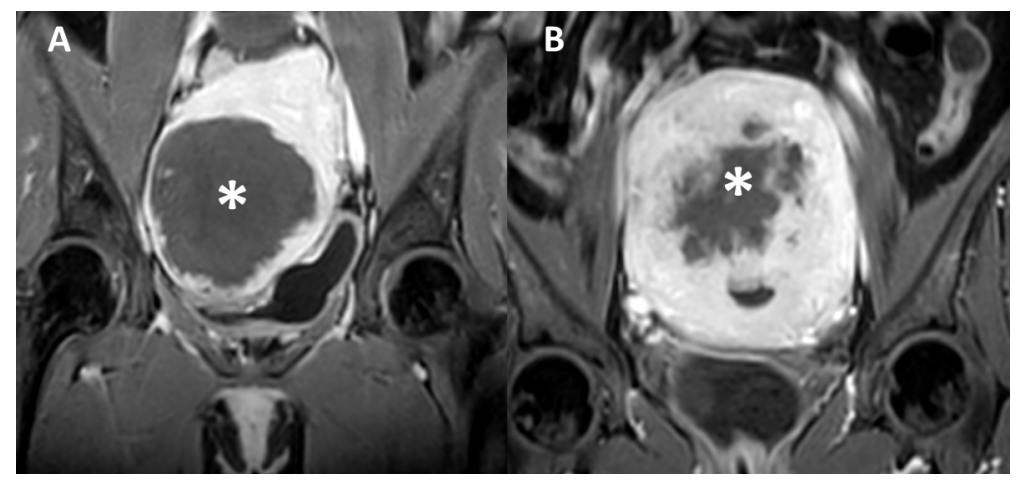

Figure 7 Coronal contrast enhancement TIW images after focused ultrasound surgery show (A) necrotic uterine fibroid (white asterisk) and (B) necrotic adenomyosis (white asterisk).
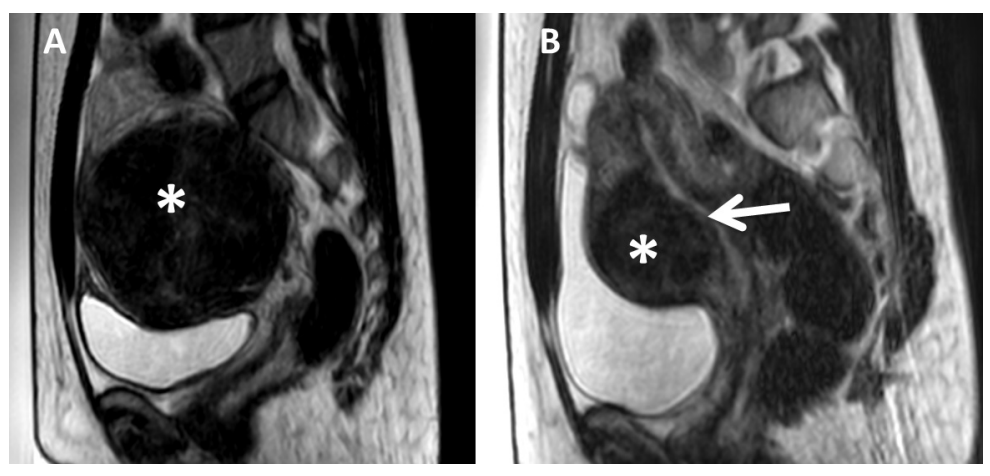

Figure 8 Sagittal T2W images show (A) intramural uterine fibroid (white asterisk) before treatment and (B) intramural uterine fibroid protruding into uterine cavity (white arrow) after 6 months of focused ultrasound surgery.

submucosal uterine fibroid. Consequently, uterine fibroid's stalk will be devascularized, leading to detachment and ejaculation. Furthermore, when the endometrial layer encircling the uterine fibroid becomes thin or turns necrotic owing to ischemic effect or hormonal changes, submucosal uterine fibroids or intramural uterine fibroids shallow to endometrium might be expelled into the uterine cavity. ${ }^{21}$

\section{Expulsion to vagina}

In Wang et al's study, ten type I submucosal uterine fibroids (transmural extension $<50 \%$ ) and 68 type II submucosal uterine fibroids (transmural extension $\geq 50 \%$ ) were ablated by ultrasound-guided FUS (Figure 9) and necrotic tissues' vaginal expulsion was observed in $58 \%$ of patients. However, after 2-4 menstrual cycles, the expulsion of necrotic 


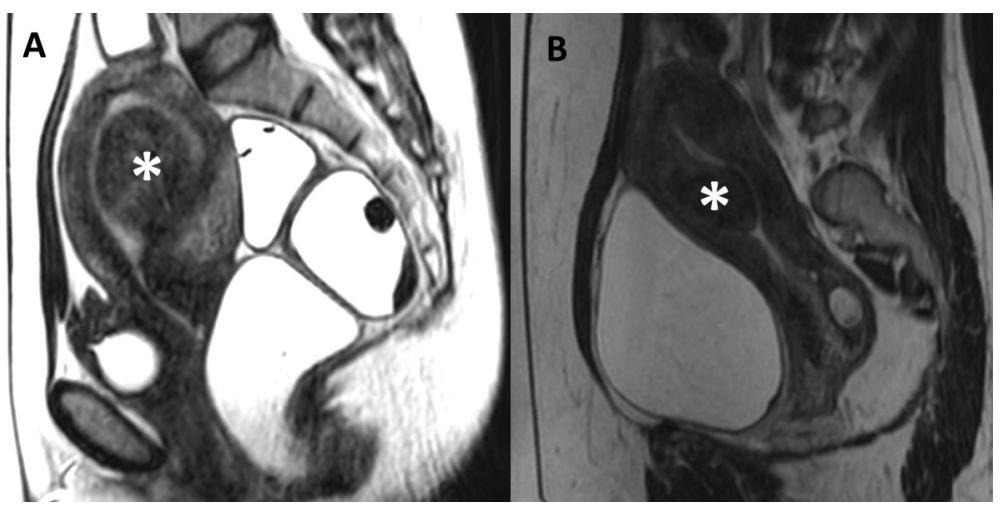

Figure 9 Sagittal T2W images show submucosal uterine fibroids (white asterisk) (A) with a transmural extension $<50 \%$ and $(\mathbf{B})$ a transmural extension $\geq 50 \%$.

tumor resolved naturally. ${ }^{22}$ Additionally, in Xie et al's study, 55 submucosal leiomyomas were divided into two groups: group 1 with 27 type I tumors and group 2 with 28 type II tumors were treated by ultrasound-guided FUS, and no significant difference was observed for the outcome of type I and type II tumors. They also observed that 12 (21.81\%) patients from both groups experienced spontaneous vaginal expulsion, and for two (3.64\%) patients, the cervix was obstructed, a complication resolved with an intravaginal forceps-assisted procedure, which was used to eliminate the obstructive necrotic fibroid tissue. ${ }^{23} \mathrm{~A}$ risk of infection of the necrotic tumor was reported; moreover, the cervix obstruction required a hysteroscopic surgery to clear up. ${ }^{21-23}$

In a case reported by Kim et al, a 38-year-old patient with uterine fibroid was admitted to the hospital due to heavy menstrual bleeding and consequent anemia and was provided MRI-guided FUS therapy. At 2-week post-therapy, the patient was readmitted with a palpable vaginal mass. MRI revealed that the ablated uterine fibroid was located in the vagina, with a narrow stem from the uterus. Two weeks after MRI examination, it was found that the tumor was not expulsed naturally and was hence excised by a hysteroscopic surgery. ${ }^{24}$ In order to prevent the patients from being anxious, clinicians should inform them about the vaginal expulsion's adverse effect after FUS therapy.

\section{Endometrial impairment}

Liu et al reported $13(0.048 \%)$ patients with abnormal vaginal discharge and bleeding. The main cause of this adverse event was damage of endometrial layer after the ablation of submucosal leiomyoma and adenomyosis. These patients were prescribed hysteroscopic surgery to excise the necrotic tissue. The symptom of abnormal discharge subsided later. ${ }^{14}$
In a study by Kim et al, the results proved that submucosal uterine fibroids deform the uterine cavity, and the endometrium around tumors becomes prone to injuries during the MRI-guided FUS ablation (Figure 10). The important factor correlated with endometrium impairment during ablation treatment is the bulging level of the submucosal uterine fibroids into the endometrial cavity. It was also observed that the endometrium impairment recovered comprehensively during the 6-month follow-up period. ${ }^{25}$

\section{Expulsion to abdominal cavity}

Pedunculated subserosal uterine fibroids are connected to the serosal layer via a narrow and small stem (Figure 11). In the past, FUS ablation for pedunculated subserosal fibroids was excluded due to the expulsion risk of tumors into the abdominal cavity after treatment. Park et al noted that there were nine pedunculated subserosal leiomyomas in 135 leiomyoma patients ablated with MRI-guided FUS. No expulsion of tumors into abdominal cavity after treatment and during follow-up period was observed. ${ }^{26}$ Likewise, in a study by Brown et al, eleven of 179 women with pedunculated leiomyomas were subjected to MRIguided FUS therapy. Two patients (1.12\%) experienced adverse events, including transient lumbar plexus stimulation and leg numbness; nonetheless, tumor expulsion to the abdominal cavity was not noticed. ${ }^{27}$ Regardless of the benefits, the adverse events of FUS ablation on pedunculated subserosal uterine fibroids remain a major concern and further studies on FUS treatment for this special type of uterine fibroids are essential.

\section{Uterine rupture}

Uterine rupture after FUS therapy was a severe adverse event observed, especially during pregnancy, owing to high rates of perinatal morbidity and even mother mortality. Kang et al noted that at 3-month post-FUS therapy, a 2-trimester 


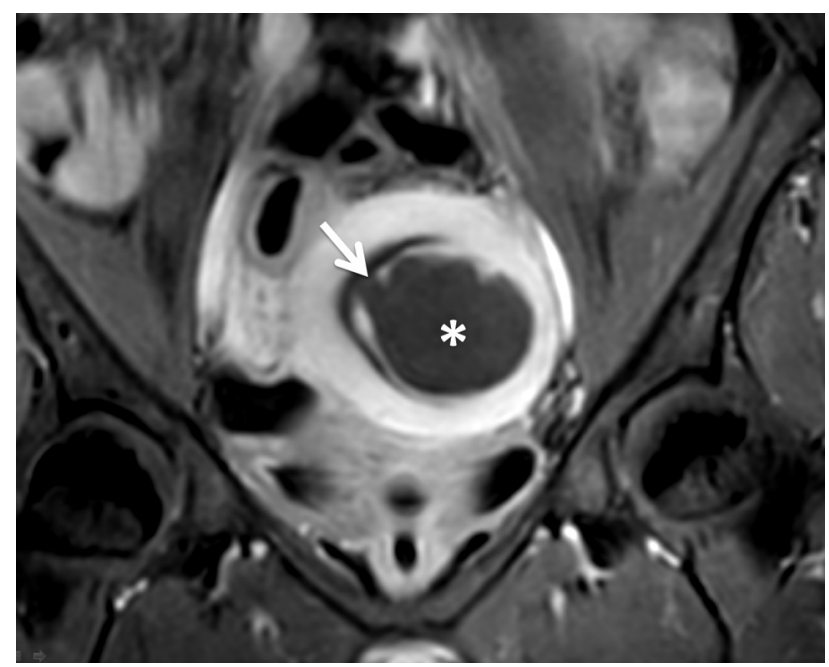

Figure 10 Coronal contrast enhancement TIW image displays a submucosal uterine fibroid (white asterisk) with spontaneous endometrial damage (white arrow) after focused ultrasound surgery.

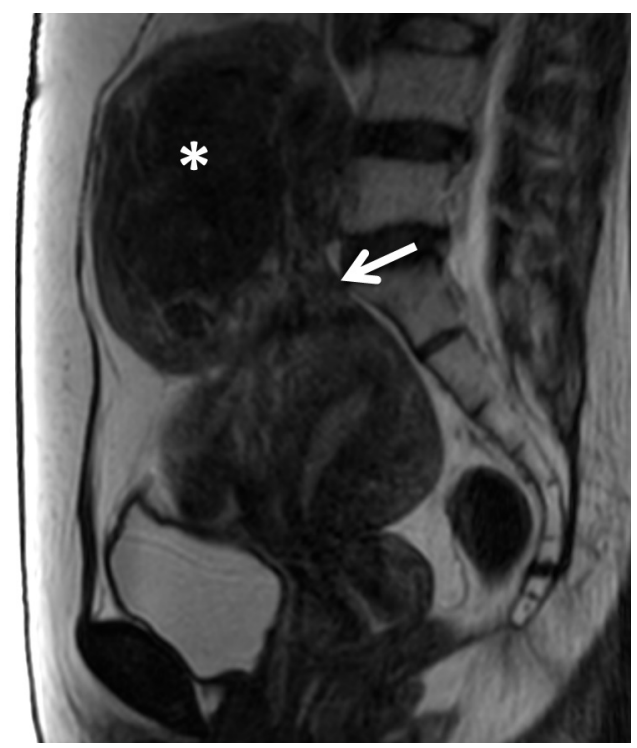

Figure II Sagittal T2W image shows subserosal uterine fibroid (white asterisk) with a small stalk (white arrow).

pregnant patient presented a uterine rupture. ${ }^{28} \mathrm{As}$ well, in a case reported by $\mathrm{Li}$ et al, a 38-week pregnancy with severe abdominal pain was referred to the hospital. The historical examination showed that the patient had a uterine fibroid over $8 \mathrm{~cm}$ in size and had undergone FUS therapy at 20 months prior to the pregnancy. A cesarean operation was carried out promptly to deliver the fetus after the stagnation of the fetal heart rate. In addition, a uterine rupture was uncovered during surgery. ${ }^{29}$ Qin et al suggested that 12 months after FUS therapy was the appropriate time to become pregnant. ${ }^{30}$ Owing to the uncertain risk of uterine rupture, large-scale investigation with long-term follow-up is crucial.

\section{Placenta accreta, placenta previa, and miscarriage}

No studies have been conducted on the relationship between miscarriage, placenta accreta, and placenta previa with FUS treatment. Nevertheless, miscarriage, placenta accreta, and placenta previa should be classified as adverse events of FUS treatment. ${ }^{6,14,19}$ Because placenta accreta and placenta previa are problematic conditions during pregnancy and delivery, we recommend that studies in the future should be conducted to determine the correlation between miscarriage, placenta previa, placenta accreta, and FUS treatment in order to update all clinicians and patients.

\section{Adverse events related to adjacent organs \\ Bladder and ureter}

According to the Maude database, bladder wall ulceration was diagnosed by an urologist at 2-week post-FUS treatment. The adverse event was completely resolved by hemostatic medication. ${ }^{19}$ In addition, Chen et al found that $52(0.52 \%)$ of 9,988 patients complained of odynuria and/or hematuria after therapy. ${ }^{13}$ Liu et al also noted that hematuria occurred in $24(0.088 \%)$ of 27,053 patients after treatment. ${ }^{14}$ These adverse events were resolved naturally without any interventional medications. They also found that urinary retention was observed in eight $(0.029 \%)$ patients with retroflexed uterus, owing to the bladder being excessively filled with normal saline for manipulating bowel loops out of the treatment window. ${ }^{14}$ Keserci et al and Liu et al reported that one $(1.35 \%)$ patient of 74 patients and four $(0.015 \%)$ patients of 27,053 patients, respectively, suffered from cystitis related to Foley catheter technique. ${ }^{9,14}$ This adverse event was eliminated completely by administering antibiotic medication within 3-7 days.

Liu et al also noted that one $(0.003 \%)$ patient with a large cervical uterine fibroid suffered from hydronephrosis of the left kidney after treatment, because the left ureter was compressed by the treated edematous leiomyoma with a diameter of over $10 \mathrm{~cm} \cdot{ }^{14}$

\section{Bowel loops}

The absorption and dispersion of ultrasonic energy of hard elements and gas bubbles inside bowel loops might inevitably cause thermal damage, even bowel burns. As stated in the FDA Maude database, two cases of bowel perforation were observed post-FUS treatment owing to the undetected uterine motion or patient movement during FUS ablation, which led to the sonication of the bowel loop, resulting in bowel 
perforation. These patients were treated through anastomosis for bowel repairment. ${ }^{19}$

Correspondingly, in a case study by Hwang et al, a 43-year-old patient with a history of menorrhagia and pelvic tension underwent FUS for ablating six uterine intramural fibroids. After the ablation therapy, the patient experienced spontaneous abdominal distress. The adverse symptom was resolved after the indication of an anti-inflammatory medication. However, the abdominal pain worsened at 14-day postFUS ablation and was not cured by painkillers. The patient was readmitted to the FUS unit for further evaluation on 29-day post-FUS ablation. CT supported the diagnosis of peritonitis and pneumoperitoneum. During operation, surgeons found a $1.5 \mathrm{~cm}$ perforation of the colon. ${ }^{18}$

Ko et al reported that a 46-year-old patient with a symptomatic adenomyosis lesion located on the posterior wall of uterus underwent ultrasound-guided FUS treatment. At 8-day post-treatment, the patient experienced severe pain in the abdomen in conjunction with peritonitis symptoms. CT examination manifested that there was free gas surrounding the stomach. During operation, surgeons found a $1 \mathrm{~cm}$ necrotic lesion at an inflammatory small bowel located at 40 $\mathrm{cm}$ from the ileocaecal valve. ${ }^{31}$ In a study by Liu et al, they found bowel injuries in four $(0.015 \%)$ patients (three patients with leiomyoma and one patient with adenomyosis). They also observed that the main cause of this severe complication was the appearance of bowel loops inside the acoustic pathway and the sonication outside of tumors. The bowel perforations occurred after 4- to 12-day post-treatment, and all patients were given a surgery for bowel repairment. In a study by Chen et al, two $(0.02 \%)$ cases of intestinal perforation were found at 10- and 12-day post-FUS treatment. The probable cause of this adverse event was calcified leiomyoma, which dispersed sonication energy to the adjacent bowel loops and sonication energy of the tissue outside of the uterine wall. It was also concluded that intestinal perforation after FUS ablation is generally delayed and appears at 10-day post-FUS treatment or later, and the symptoms are not typical to acute bowel perforation. ${ }^{13}$ When focusing energy on the region near the perimetrium, the clinicians need to be careful and should optimize treatment strategy to avoid bowel injury. Also, to yield the non-bowel treatment window for patients during ablation, bladder filling and rectum filling maneuver technique should be managed (Figure 12). ${ }^{15,16}$

\section{Ovary}

One of the most important organs close to the uterine fibroid and/or adenomyosis lesions is the ovary. Given the concern about FUS therapy's negative impact on ovarian function, some studies have proved that there are no side effects on ovarian function by the preservation of serum anti-Müllerian hormone and the conservation of serum follicle-stimulating hormone, luteinizing hormone, and estradiol at 6- and 12-month follow-up. ${ }^{32-34}$ Nevertheless, no long-term followup studies have focused on the changes in these hormones and ovarian volume. Therefore, further research on the FUS effect on the ovarian functions is still required.

\section{Adverse events related to whole body Nausea}

Nausea and vomiting are common and slight adverse events of FUS treatment. During the ablation procedure and after

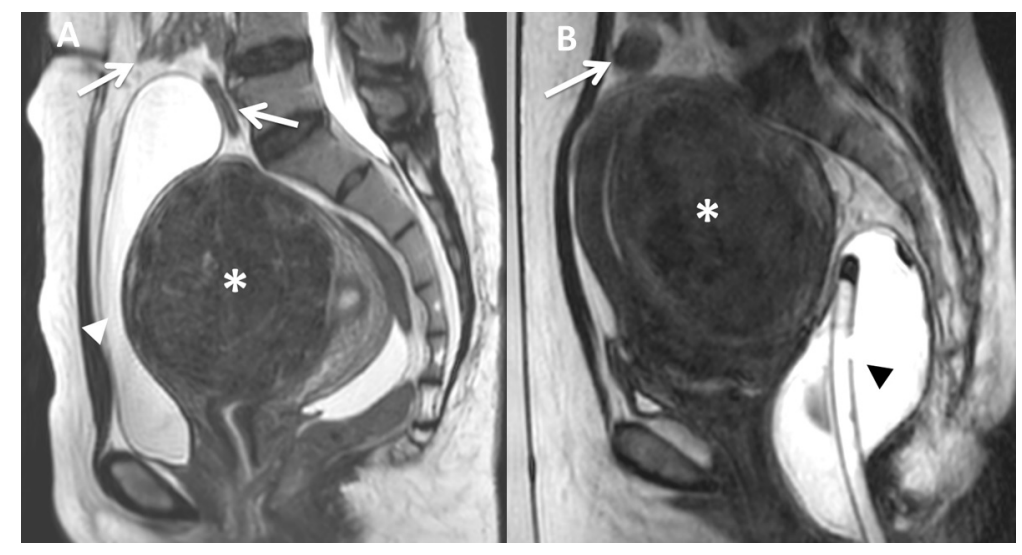

Figure 12 Sagittal T2W images show (A) uterine fibroid on the anterior wall of the retroverted uterus (white asterisk) and a bladder filled with $400 \mathrm{~mL}$ normal saline (white arrow head) generated bowel loops out of the treatment window (white arrow); (B) uterine fibroid on the posterior wall of anteverted uterus (white asterisk) and rectum filled with $200 \mathrm{~mL}$ ultrasound gel (black arrow head) produced bowel loops out of the treatment window (white arrow). 
therapy, some drugs, such as non-steroid painkiller, fentanyl citrate, oxytocin, and gadolinium-based contrast agents are used, which can produce side effects such as nausea and vomiting. . $^{6,9,10,13,14}$

\section{Deep vein thrombosis and blood disorder}

In an evidence-based study of MRI-guided FUS by Pron, it was found that the risk of deep vein thrombosis increases due to long ablation duration of prone position, especially in the case of a great volume of uterine fibroids or adenomyosis. ${ }^{6-8}$ Liu et al noted that two patients suffered from deep vein thrombosis after ablation. The patients were prescribed medications, following which they returned to normal. In addition, they also observed that one patient suffered from thrombocytopenia after ablation with unclear cause. ${ }^{14}$ Thus, it is crucial to investigate the blood coagulation function of patients prior to FUS treatment.

\section{Tumor lysis syndrome and renal impairment}

Chen et al found three $(0.03 \%)$ cases of acute renal failure after FUS ablation; however, the reasonable cause was not mentioned in the report. ${ }^{13}$ In a case reported by Park et al, a 35 -year-old patient with a $14 \times 10 \mathrm{~cm}$ leiomyoma underwent FUS ablation. After 5 days of treatment, the patient suffered from fever and pain in the back and abdomen. Blood test unveiled that the patient was suffering from hyperuricemia, hyperphosphatemia, slight metabolic acidosis, and acute kidney insufficiency after FUS ablation. The plasma concentrations of urea nitrogen, creatinine, uric acid, calcium, and phosphorus at 5-day post-treatment were $77 \mathrm{mg} / \mathrm{dL}$, $4.6 \mathrm{mg} / \mathrm{dL}, 11.3 \mathrm{mg} / \mathrm{dL}, 7.6 \mathrm{mg} / \mathrm{dL}$, and $5.0 \mathrm{mg} / \mathrm{dL}$ and at 14-day post-treatment were $7 \mathrm{mg} / \mathrm{dL} 1.0 \mathrm{mg} / \mathrm{dL}, 4.9 \mathrm{mg} / \mathrm{dL}$, $8.1 \mathrm{mg} / \mathrm{dL}, 3.2 \mathrm{mg} / \mathrm{dL}$, respectively. ${ }^{35}$ It was concluded that necrotic volume caused by chemotherapy, radiation therapy,
FUS, and radio-frequency ablation, among others, mediated to release cellular substances such as uric acid, potassium, and phosphorus, which can lead to acute kidney injury and even death. ${ }^{36,37}$ Liu et al reported that four $(0.015 \%)$ patients with uterine fibroids experienced acute kidney failure after FUS treatment. For three of them, kidney function returned to normal level after treatment with medications. However, one patient had to undergo hemodialysis; nonetheless, the serum creatine level was still about $200 \mu \mathrm{mol} / \mathrm{L} .{ }^{14}$ Even though the incidence rate of acute renal failure post-FUS therapy is not high, there is a possibility of this severe adverse event. Thus, kidney functions should be investigated cautiously prior to and after FUS ablation. The use of antibiotics and non-steroid drugs after treatment should be considered owing to increasing risk of acute kidney injury. In addition, to avoid this severe adverse event for patients with big tumors, the clinicians should utilize medication such as gonadotropin-releasing hormone agonists prior to the FUS treatment to alleviate the volume of the leiomyoma (Figure 13) and adenomyosis (Figure 14). ${ }^{38,39}$

\section{Death}

In a case reported by Bhise et al, a diffuse adenomyosis patient received MRI-guided FUS treatment. However, when the patient tried to stand post-treatment, she abruptly collapsed. In spite of revival efforts, the patient died on the same day at 4-hour post-treatment. Forensic investigation revealed that an abdominal wall was distended. Also, approximately $3 \mathrm{~L}$ of blood was present in the abdominal cavity. Likewise, the right uterine artery and right ovarian artery were dissected. The cause of death was concluded to be hypovolemic shock due to hemorrhage following rupture of right uterine artery and right ovarian artery. Those arteries appeared in the sonication pathway of FUS ablation; thus,

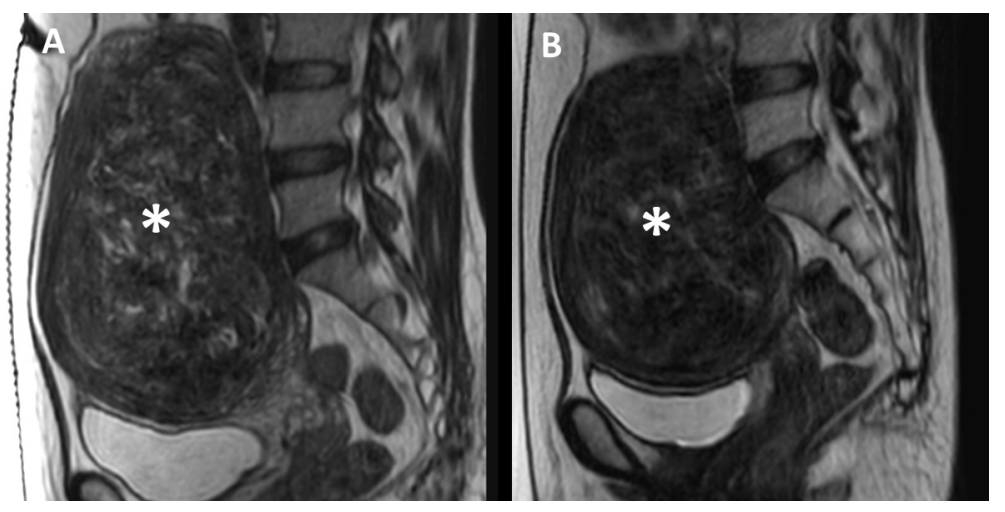

Figure 13 Sagittal T2W images show the uterine fibroid (white asterisk) (A) prior to GnRH agonist therapy and (B) after 3 months of GnRH agonist therapy; the volume of uterine fibroid reduced up to $38 \%$. 
FUS generated the damage of the intima leading to arterial dissection and rupture..$^{40}$

According to the Maude database, there was one case of heart attack spontaneously after FUS ablation. The patient was resuscitated successfully. Nonetheless, the patient experienced a second heart attack, and the revival effort failed. Blood test manifested that the hemoglobin level prior to treatment and at the time of heart attack was $10.8 \mathrm{mg} / \mathrm{dL}$ and $3 \mathrm{mg} /$ $\mathrm{dL}$, respectively. The probable cause of death was not found, and no malfunction was detected in the FUS machine. ${ }^{19}$ In order to avoid heart adverse event, electrocardiography and/ or echocardiography should be assessed delicately prior to treatment.

\section{Ablation of leiomyosarcoma}

Samuel et al stated that leiomyosarcoma and leiomyoma might manifest a high similarity in imaging characteristics, leading to a misdiagnosis (Figure 15). ${ }^{41}$ The relevant treatment for leiomyosarcoma is hysterectomy for ensuring long-term prognosis; hence, FUS treatment for the leiomyosarcoma was regarded as a severe adverse event. In their report, a 47-year-old patient with a uterine tumor over 10 $\mathrm{cm}$ in size wished to receive FUS therapy. The MRI findings showed heterogeneous signal intensity on $\mathrm{T} 2 \mathrm{~W}$ images and both $\mathrm{T} 1 \mathrm{~W}$ images prior to and after contrast enhancement. There was hemorrhage and necrosis inside the tumor. The clinician advised surgery instead of FUS ablation due to the atypical nature of the uterine tumor. Histopathological result of tumor showed leiomyosarcoma tissues. ${ }^{41}$

According to the Maude database, one voluntary patient with uterine leiomyosarcoma was misdiagnosed with uterine fibroid, which was found after 1 year of FUS treatment. Early stage uterine cancer was diagnosed and hysterectomy was indicated. ${ }^{19}$

In a report by Fukunishi et al, a 40-year-old patient with a $9 \mathrm{~cm}$ uterine fibroid underwent MRI-guided FUS therapy. At 6-month post-ablation, the symptoms aggravated. MRI images exhibited that the uterine fibroid volume had not reduced. A morcellation via laparoscopic surgery was exploited. Histopathologic results manifested that the right

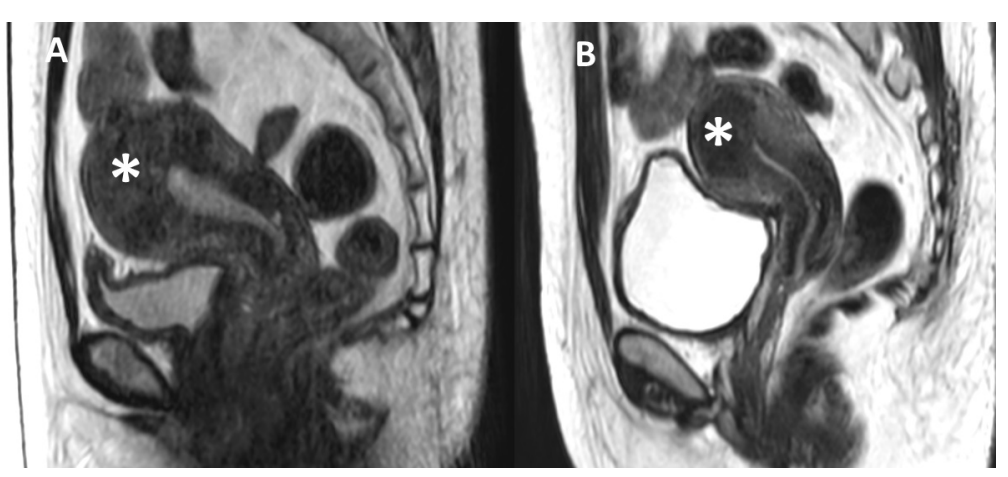

Figure I4 Sagittal T2W images show adenomyosis (white asterisk) (A) prior to GnRH agonist therapy and (B) after 3 months of GnRH agonist therapy; the volume of adenomyosis reduced up to $26 \%$.

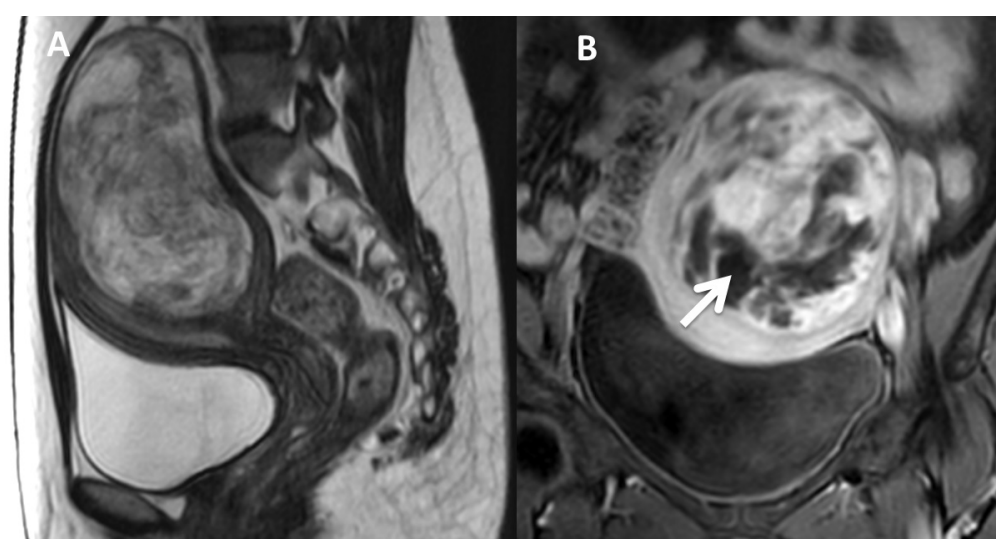

Figure 15 Sagittal T2W image (A) and coronal contrast enhancement TIW image (B) of a uterine leiomyosarcoma patient display a heterogeneous signal intensity tumor with partial necrosis (white arrow). 
diagnosis in this case was a leiomyosarcoma tumor. Patient was advised a radical hysterectomy to completely eliminate the tumor tissue. ${ }^{42}$ In some previous studies, MRI was more accurate than other modalities in differentiating malignant and benign uterine tumors. ${ }^{43,44}$ Therefore, MRI screening prior to FUS treatment should be managed carefully to avoid postponing hysterectomy for leiomyosarcoma.

\section{Ultrasound-guided FUS versus MRI- guided FUS}

Two real-time modalities have been exploited to guide FUS ablation: ultrasound or MRI. In terms of the costeffectiveness, space consumption, susceptibility to motion, and real-time ability, ultrasound-guided FUS is superior to MRI-guided FUS. Nonetheless, thermometry during FUS treatment, closed-loop feedback control, tissue contrast, images of adjacent organs surrounding uterus, and field of view of MRI-guided FUS are better than ultrasound-guided FUS. ${ }^{45}$ Owing to different capabilities of image-guided methods and experience of FUS users, there are reasonable differences in incidence of adverse events of each center and study. However, currently there are no studies comparing the incidence of each adverse event between two methods focused on the pelvic applications.

\section{Conclusion}

Despite the potential and efficacy of FUS therapy, all the adverse events discussed in this article should be considered cautiously by clinicians to ensure FUS treatment's safety profile and effective outcomes for patients with uterine fibroids and adenomyosis. Also, patients have priority to acknowledge all of the possible adverse events and thus should be informed in order to achieve a comprehensive agreement prior to FUS treatment. Further studies focused on the pros and cons, efficacy, acceptability, adverse events, and safety should be managed to not solely update FUS users and patients but also establish better protocol for FUS treatment.

\section{Ethical statement}

Written informed consent was obtained from all patients for using their images in this article.

\section{Disclosure}

The authors report no conflicts of interest in this work.

\section{References}

1. Stewart EA. Clinical practice. Uterine Fibroids. N Engl J Med. 2015; 372(17):1646-1655.
2. Ferrari F, Arrigoni F, Miccoli A, et al. Effectiveness of magnetic resonance-guided focused ultrasound surgery (MRgFUS). Radiol Med. 2016;121(2):153-161.

3. Coakley FV, Foster BR, Farsad K, et al. Pelvic applications of MR-guided high intensity focused ultrasound. Abdom Imaging. 2013;38(5):1120-1129.

4. Ellens N, Hynynen K. Simulation study of the effects of near- and farfield heating during focused ultrasound uterine fibroid ablation using an electronically focused phased array: A theoretical analysis of patient safety. Med Phys. 2014;41(7):072902.

5. Zhang X, Li K, Xie B, He M, He J, Zhang L. Effective ablation therapy of adenomyosis with ultrasound-guided high-intensity focused ultrasound. Int J Gynaecol Obstet. 2014;124(3):207-211.

6. Pron G. Magnetic Resonance-Guided High-Intensity Focused Ultrasound (MRgHIFU) Treatment of Symptomatic Uterine Fibroids: An Evidence-Based Analysis. Ont Health Technol Assess Ser. 2015;15(4):1-86.

7. Duc NM, Huy HQ. Effect of magnetic resonance imaging characteristics on uterine fibroid treatment. Reports in medical imaging. 2018;11:1-8.

8. Duc NM, Huy HQ. Influences of screening magnetic resonance imaging parameters on high-intensity focused ultrasound outcome for adenomyosis. Reports in medical imaging. 2018;11:9-14.

9. Keserci B, Duc NM. The role of T1 perfusion-based classification in magnetic resonance-guided high-intensity focused ultrasound ablation of uterine fibroids. Eur Radiol. 2017;27(12):5299-5308.

10. Keserci B, Duc NM. The role of T1 perfusion-based classification in predicting the outcome of magnetic resonance-guided high-intensity focused ultrasound treatment of adenomyosis. Int J Hyperthermia. 2018;34(3):306-314.

11. Leon-Villapalos J, Kaniorou-Larai M, Dziewulski P. Full thickness abdominal burn following magnetic resonance guided focused ultrasound therapy. Burns. 2005;31(8):1054-1055.

12. Keserci B, Duc NM. Volumetric magnetic resonance-guided high-intensity focused ultrasound ablation of uterine fibroids through abdominal scars: the impact of a scar patch on therapeutic efficacy and adverse effects. J Ther Ultrasound. 2017;5:22.

13. Chen J, Chen W, Zhang L, et al. Safety of ultrasound-guided ultrasound ablation for uterine fibroids and adenomyosis: A review of 9988 cases. Ultrason Sonochem. 2015;27:671-676.

14. Liu Y, Zhang WW, He M, et al. Adverse effect analysis of high-intensity focused ultrasound in the treatment of benign uterine diseases. Int $J$ Hyperthermia. 2018:1-6.

15. Kim YS, Bae DS, Park MJ, et al. Techniques to expand patient selection for MRI-guided high-intensity focused ultrasound ablation of uterine fibroids. AJR Am J Roentgenol. 2014;202(2):443-451.

16. Duc NM, Huy HQ. High-intensity focused ultrasound of uterine fibroids and adenomyosis: Maneuver technique for bowel loops located inside the treatment window. Imaging Med. 2018;10(2):29-31.

17. Hindley J, Gedroyc WM, Regan L, et al. MRI guidance of focused ultrasound therapy of uterine fibroids: early results. AJR Am J Roentgenol. 2004;183(6):1713-1719.

18. Hwang DW, Song HS, Kim HS, Chun KC, Koh JW, Kim YA. Delayed intestinal perforation and vertebral osteomyelitis after high-intensity focused ultrasound treatment for uterine leiomyoma. Obstet Gynecol Sci. 2017;60(5):490-493.

19. U.S. Food and Drug Administration [webpage on the Internet]. MAUDE:Manufacturer and user facility device experience. MAUDE adverse eventreport: Insightec, 1td. EXABLATE 2000 MRGFUS; 2012. Available from: www.accessdata.fda.gov/scripts/cdrh/cfdocs/cf-maude/ search.cfm. Accessed 18 May 2018.

20. Kim HK, Kim D, Lee MK, et al. Three cases of complications after high-intensity focused ultrasound treatment in unmarried women. Obstet Gynecol Sci. 2015;58(6):542-546.

21. Jeong JH, Hong GP, Kim YR, et al. Expulsion of Fibroids to the Endometrial Cavity after Magnetic Resonance Imaging-guided High Intensity Focused Ultrasound Surgery (MRgFUS) Treatment of Intramural Uterine Fibroids. J Menopausal Med. 2016;22(3):139-145. 
22. Wang W, Wang Y, Wang T, Wang J, Wang L, Tang J. Safety and efficacy of US-guided high-intensity focused ultrasound for treatment of submucosal fibroids. Eur Radiol. 2012;22(11):2553-2558.

23. Xie B, Zhang C, Xiong C, He J, Huang G, Zhang L. High intensity focused ultrasound ablation for submucosal fibroids: A comparison between type I and type II. Int J Hyperthermia. 2015;31(6):593-599.

24. Kim KA, Yoon SW, Yoon BS, Park CT, Kim SH, Lee JT. Spontaneous vaginal expulsion of uterine myoma after magnetic resonance-guided focused ultrasound surgery. J Minim Invasive Gynecol. 2011;18(1): $131-134$.

25. Kim YS, Kim TJ, Lim HK, et al. Preservation of the endometrial enhancement after magnetic resonance imaging-guided high-intensity focused ultrasound ablation of submucosal uterine fibroids. Eur Radiol. 2017;27(9):3956-3965.

26. Park H, Yoon SW, Kim KA, Jung Kim D, Jung SG. Magnetic resonance imaging-guided focused ultrasound treatment of pedunculated subserosal uterine fibroids: a preliminary report. J Vasc Interv Radiol. 2012;23(12):1589-1593.

27. Brown DL, Hesley GK, Gorny KR, Stewart EA. Safety of MR-guided focused ultrasound treatment of pedunculated subserosal uterine leiomyomas. J Ther Ultrasound. 2013;1:19.

28. Kang YD, Park CH. Spontaneous Uterine Rupture during Second Trimester Pregnancy after High-intensity Focused Ultrasound. Korean J Perinatol. 2015;26(4):373-376.

29. Li XW, Liang MY, Wang JL, Wang DP. Spontaneous Uterine Rupture during Late Pregnancy after High-intensity Focused Ultrasound. Chin Med J (Engl). 2015;128(10):1419.

30. Qin J, Chen JY, Zhao WP, Hu L, Chen WZ, Wang ZB. Outcome of unintended pregnancy after ultrasound-guided high-intensity focused ultrasound ablation of uterine fibroids. Int J Gynaecol Obstet. 2012;117(3):273-277.

31. Ko JKY, Seto MTY, Cheung VYT. Thermal bowel injury after ultrasound-guided high-intensity focused ultrasound treatment of uterine adenomyosis. Ultrasound Obstet Gynecol. 2018;52(2):282-283.

32. Lee JS, Hong GY, Lee KH, Kim TE. Changes in anti-müllerian hormone levels as a biomarker for ovarian reserve after ultrasound-guided high-intensity focused ultrasound treatment of adenomyosis and uterine fibroid. BJOG. 2017;124 Suppl 3:18-22.
33. Cheung VY, Lam TP, Jenkins CR, Cheung GK, Chan SS, Choi WK. Ovarian Reserve After Ultrasound-Guided High-Intensity Focused Ultrasound for Uterine Fibroids: Preliminary Experience. J Obstet Gynaecol Can. 2016;38(4):357-361.

34. Chen H, Huang Y, Zhang WX, Sx L, Chen ZX. Study of influence on ovarian function by HIFU and surgical treatment for uterine fibroids. Chin J Obstet Gynecol Pediatr. 2012;8(4):468-470.

35. Park J-H, Yang I-M, Kim Y-H, Yu SJ, Lee JS, Kim DH. High Intensity Focused Ultrasound Treatment-Induced Tumor Lysis Syndrome in Uterine Myoma Patient. Soonchunhyang Med Sci (SMS). 2015;21(2):99-101.

36. Mirrakhimov AE, Ali AM, Khan M, Barbaryan A. Tumor Lysis Syndrome in Solid Tumors: An up to Date Review of the Literature. Rare Tumors. 2014;6(2):5389.

37. Wilson FP, Berns JS. Onco-nephrology: tumor lysis syndrome. Clin J Am Soc Nephrol. 2012;7(10):1730-1739.

38. Smart OC, Hindley JT, Regan L, Gedroyc WM. Magnetic resonance guided focused ultrasound surgery of uterine fibroids--the tissue effects of GnRH agonist pre-treatment. Eur J Radiol. 2006;59(2):163-167.

39. Guo Y, Duan H, Cheng J, Zhang Y. Gonadotrophin-releasing hormone agonist combined with high-intensity focused ultrasound ablation for adenomyosis: a clinical study. BJOG. 2017;124 Suppl 3:7-11.

40. Bhise SS, Chavan GS, Nanandkar SD, Chikhalkar BG, Thube HR. First Reported Death in India during MRgFUS: A Case Report. Journal of Indian Academy of Forensic Medicine. 2015;37(2):204-206.

41. Samuel A, Fennessy FM, Tempany CM, Stewart EA. Avoiding treatment of leiomyosarcomas: the role of magnetic resonance in focused ultrasound surgery. Fertil Steril. 2008;90(3):850.e9-12.

42. Fukunishi H, Funaki K, Ikuma K, et al. Unsuspected uterine leiomyosarcoma: magnetic resonance imaging findings before and after focused ultrasound surgery. Int J Gynecol Cancer. 2007;17(3):724-728.

43. Schwartz LB, Zawin M, Carcangiu ML, Lange R, Mccarthy S. Does pelvic magnetic resonance imaging differentiate among the histologic subtypes of uterine leiomyomata? Fertil Steril. 1998;70(3):580-587.

44. Tanaka YO, Nishida M, Tsunoda H, Okamoto Y, Yoshikawa H. Smooth muscle tumors of uncertain malignant potential and leiomyosarcomas of the uterus: MR findings. J Magn Reson Imaging. 2004;20(6):998-1007.

45. Kim Y-S. Clinical application of high-intensity focused ultrasound ablation for uterine fibroids. Biomed Eng Lett. 2017;7(2):99-105.
Reports in Medical Imaging

\section{Publish your work in this journal}

Reports in Medical Imaging is an international, peer-reviewed, open access journal publishing original research, reports, reviews and commentaries on all areas of medical imaging. The manuscript management system is completely online and includes a very quick and fair peer-review system, which is all easy to use.

\section{Dovepress}

Visit http://www.dovepress.com/testimonials.php to read real quotes from published authors. 